\title{
Estudo comparativo das medidas manual e digital da fenda palpebral
}

\author{
A comparative study of the manual and digital measurements of the palpebral fissure
}

Tânia Pereira Nunes ${ }^{1}$

Thomaz Fracon de Oliveira ${ }^{2}$

Suzana Matayoshi ${ }^{3}$

\section{RESUMO}

Objetivo: Comparar a medida manual do comprimento vertical da fenda palpebral e sua medida obtida por meio de processamento computadorizado de imagens de indivíduos sem alterações palpebrais. Métodos: Foram analisadas 102 fendas palpebrais de 51 indivíduos normais. A faixa etária variou entre 17 e 84 anos, sendo 25 (49\%) do sexo masculino e $26(51 \%)$ do sexo feminino. Resultados: Não houve diferença estatisticamente significativa entre as medidas manuais da fenda palpebral e as obtidas por meio de processamento computadorizado de imagens. Também não se observou diferença entre ambos os sexos e a faixa etária. Conclusões: Os resultados obtidos com estes dois métodos podem ser comparados entre si devida à equivalência estatística das medidas.

Descritores: Pálpebras/anatomia \& histologia; Doenças palpebrais; Medidas; Processa mento de imagem assistida por computador; Estudo comparativo

\section{INTRODUÇÃO}

A fenda palpebral (FP) pode ser definida como um espaço delimitado pelas pálpebras superior e inferior. Sua avaliação normalmente é realizada quando o indivíduo está em estado de alerta e com os olhos em posição primária do olhar (PPO). O equilíbrio entre os músculos retratores, aqueles responsáveis pela abertura da FP, e os músculos protractores, aqueles que realizam seu fechamento, é responsável pela medida do comprimento vertical da $\mathrm{FP}^{(1)}$.

A medida do comprimento vertical da FP é um parâmetro importante nas assimetrias palpebrais $^{(1-2)}$. Os dados clínicos relacionados às suas dimensões em geral são obtidos com instrumentos simples, como a régua milimetrada ${ }^{(3)}$; porém, técnicas de processamento digital de imagens têm sido utilizadas para o estudo do contorno palpebral, fornecendo importantes dados, como posicionamento e curvatura palpebral, área e angulação da $\mathrm{FP}^{(4-6)}$.

O objetivo deste trabalho é comparar a medida manual do comprimento vertical da FP e sua medida obtida por meio de processamento computadorizado de imagens de indivíduos sem alterações palpebrais.

\section{MÉTODOS}

Foram analisadas as medidas manuais do comprimento vertical da FP de 51 indivíduos sem alterações palpebrais, e as medidas obtidas por meio de processamento computadorizado de imagens. Foram tomados os devidos cuidados para selecionar pessoas que não apresentassem doenças sistêmicas ou oftalmológicas que pudessem alterar a FP.

Optou-se pela divisão dos indivíduos em 4 grupos: os grupos 1 e 2 (G1 - 
G2) eram formados por indivíduos do sexo masculino; o G1 apresentava faixa etária inferior a 50 anos de idade, e o G2 com idade acima de 50 anos. Os grupos 3 e 4 (G3 - G4) eram formados por indivíduos do sexo feminino; o G3 apresentava faixa etária inferior a 50 anos de idade e o G4 com idade acima de 50 anos.

Foram analisados os dois olhos de cada paciente, ou seja, $102 \mathrm{FP}$

A medida manual da altura da FP foi realizada bilateralmente com os olhos do paciente na mesma altura que os do examinador, tendo sido utilizada uma régua milimétrica para a medição. A régua era posicionada verticalmente e tomava como parâmetro o eixo pupilar ${ }^{(1,3)}$. Todas medidas foram realizadas pelo mesmo examinador.

O registro das imagens da FP foi efetuado através de uma câmara digital Cyber-shot, modelo: DSC-S75/S85, Sony. A captação da imagem era feita a uma distância de $50 \mathrm{~cm}$ com a cabeça do paciente posicionada em um apoio (Fig. 1) e iluminação artificial, ambiente da sala do ambulatório. Foi fixada uma régua próxima da região frontal do paciente, no sentido horizontal, possibilitando assim padronizar as medidas de todas as imagens. O paciente permanecia em PPO quando era então captada a imagem. As imagens eram transferidas para um microcomputador equipado com um programa de captação e edição de imagens: Scion Image Frame Grabber ${ }^{(6)}$ (Fig. 2).

Análises estatísticas entre os grupos foram feitas com o Kruskal-Wallis test.

Aceitou-se um valor de $\mathrm{p}<0,05$ como significativo.

\section{RESULTADOS}

A faixa etária dos indivíduos variou entre 17 e 84 anos (média: 50,5 anos), sendo 25 pacientes (49\%) do sexo masculino e $26(51 \%)$ do sexo feminino.

O G1 apresentou faixa etária entre 23 e 41 anos (média: 32 anos), o G2 apresentou idade mínima de 51 e máxima de 84 anos (média: 67,5 anos). O G3 apresentou faixa etária entre 17

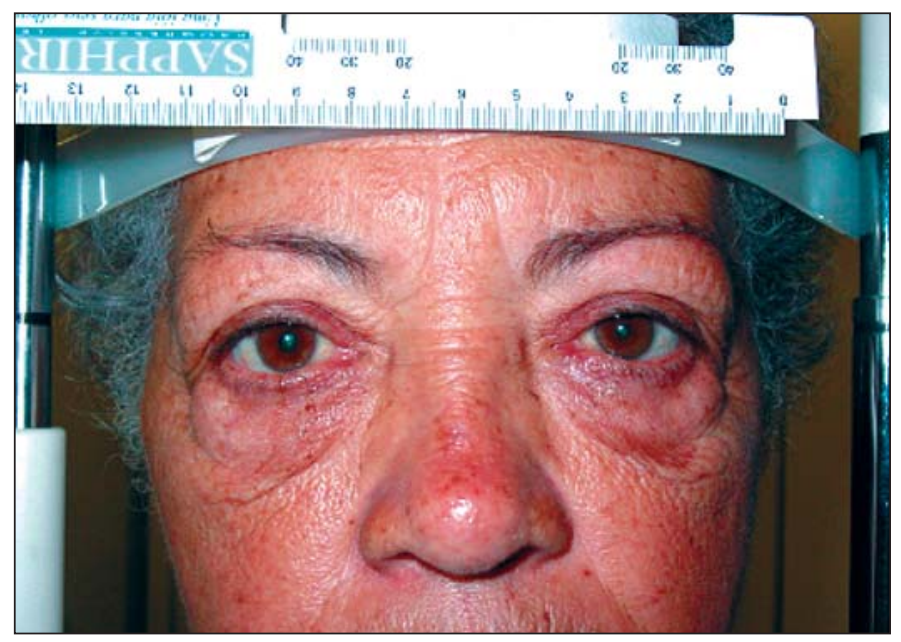

Figura 1 - Captação da imagem de uma paciente com a cabeça posicionada em um apoio

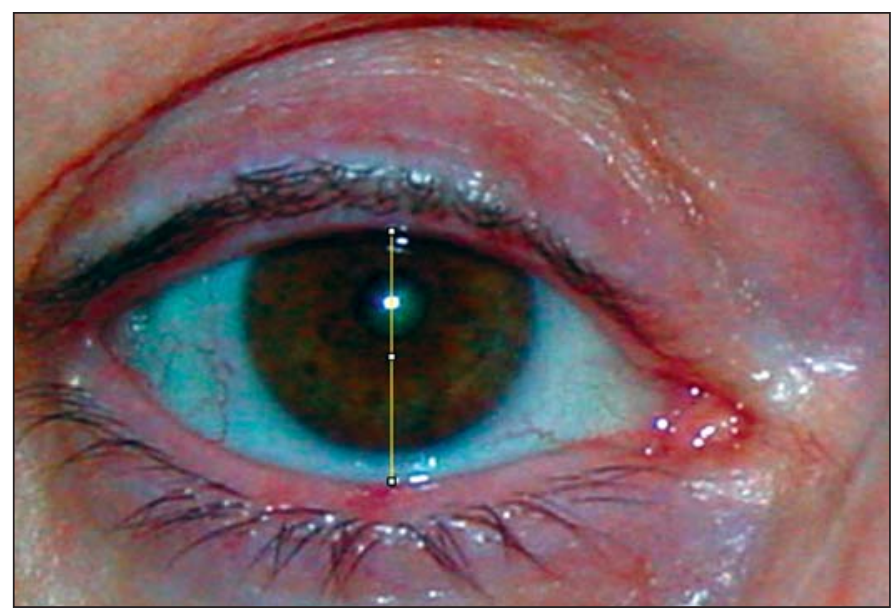

Figura 2 - Medida computadorizada da fenda palpebral

e 47 anos (média: 32 anos), e o G4, entre 54 e 80 anos (média: 67 anos) (Quadro 1).

O G1 apresentou como média do comprimento vertical da FP pela medida manual de olho direito (OD): $9,8 \mathrm{~mm}$, e de olho esquerdo (OE): 9,5 mm. As medidas obtidas por meio de processamento computadorizado de imagens foram: OD: $9,53 \mathrm{~mm}$ e OE: $9,34 \mathrm{~mm}$. A comparação entre a medida manual e a medida digital da FP em OD e OE não foi estatisticamente significante (em OD p: 0,12 e em OE p: 0,20).

O G2 apresentou como média do comprimento vertical da FP pela medida manual de olho direito (OD): $9,28 \mathrm{~mm}$, e de olho esquerdo (OE): $9,10 \mathrm{~mm}$. As medidas obtidas por meio de processamento computadorizado de imagens foram: OD: 9,38 mm e OE: $9,27 \mathrm{~mm}$. A comparação entre a medida manual e a medida digital da FP em OD e OE não foi estatisticamente significante (em OD p: 0,21 e em OE p: 0,19).

O G3 apresentou como média do comprimento vertical da FP pela medida manual de olho direito (OD): $10,6 \mathrm{~mm}$, e de olho esquerdo (OE): 10,6 mm. As medidas obtidas por meio de processamento computadorizado de imagens foram: OD: 9,98 mm e OE: $10,1 \mathrm{~mm}$. A comparação entre a medida manual e a medida digital da FP em OD e OE não foi estatisticamente significante (em OD p: 0,33 e em OE p: 0,35).

O G4 apresentou como média do comprimento vertical da FP pela medida manual de olho direito (OD): $8,94 \mathrm{~mm}$, e de olho esquerdo (OE): $8,83 \mathrm{~mm}$. As medidas obtidas por meio de pro-

\begin{tabular}{|c|c|c|}
\hline \multicolumn{3}{|c|}{ Quadro 1. Divisão dos indivíduos em 4 grupos } \\
\hline & Sexo & Faixa etária (anos) \\
\hline G1 & Masculino & $\begin{array}{c}<50 \\
\text { (Mínima: } 23 \text { Máxima: 41) }\end{array}$ \\
\hline G2 & Masculino & $\begin{array}{c}>50 \\
\text { (Mínima: } 51 \text { Máxima: } 84 \text { ) }\end{array}$ \\
\hline G3 & Feminino & $\begin{array}{c}<50 \\
\text { (Mínima: } 17 \text { Máxima: 47) }\end{array}$ \\
\hline G4 & Feminino & $\begin{array}{c}>50 \\
\text { (Mínima: } 54 \text { Máxima: } 80 \text { ) }\end{array}$ \\
\hline
\end{tabular}


cessamento computadorizado de imagens foram: OD: 8,92 mm e OE: $8,98 \mathrm{~mm}$. A comparação entre a medida manual e a medida digital da FP em OD e OE não foi estatisticamente significante (em OD p: 0,33 e OE p: 0,19). Os resultados encontram-se no quadro 2.

\section{DISCUSSÃO}

O estudo das características palpebrais, incluindo a FP, tem sido realizado há muitos anos, na tentativa de conhecer melhor a anatomia, as alterações relacionadas ao envelhecimento e as diversas afecções palpebrais.

Neste trabalho analisou-se a altura da FP em indivíduos divididos em 4 diferentes grupos, avaliaram-se separadamente indivíduos do sexo masculino e feminino e em relação à faixa etária. Os resultados mostraram valores semelhantes das medidas, tanto a realizada de forma manual, como também na medida digital. As medidas do comprimento vertical da FP foram discretamente menores nos grupos com idade acima dos 50 anos, porém não houve diferença estatisticamente significativa. Também não se observou diferença entre as medidas de OD e OE. Fox, em seu estudo com 1686 indivíduos com idade entre 10 e 60 anos, encontrou o valor médio da altura da FP de $9,24 \mathrm{~mm}^{(7)}$. Cruz e Baccega obtiveram uma média de 9,02 mm analisando 70 FP de pacientes entre 10 e 60 anos $^{(4)}$.

Alguns autores observaram que a distância entre a margem palpebral superior e o centro pupilar diminuiu discretamente com a idade, tanto em indivíduos do sexo masculino como também no sexo feminino. Porém em ambos os sexos, a distância entre o centro pupilar e margem palpebral inferior aumentou com a idade, isto pode ser explicado pela flacidez tecidual decorrente do processo de envelhecimento e ação da gravidade. Este aumento foi maior em homens que em mulheres ${ }^{(8)}$.

Com a utilização do processamento digital de imagens, as análises quantitativas foram bastante refinadas, porém quando comparadas com as medidas manuais, não apresentaram diferença estatisticamente significativa. As vantagens da técnica de processamento digital de imagens são: é um método preciso e reprodutível, o que difere da técnica manual, que necessita de uma certa experiência para ser realizada de forma correta; a medida manual da FP deve ser realizada com os olhos do paciente em PPO, o examinador deve estar na mesma altura do paciente, deve-se utilizar sempre a mesma régua

\begin{tabular}{|c|c|c|c|c|c|c|}
\hline \multicolumn{7}{|c|}{ Quadro 2. Comparação das medidas da fenda palpebral } \\
\hline & \multicolumn{6}{|c|}{ Comprimento } \\
\hline & \multicolumn{2}{|c|}{$\begin{array}{c}\text { Medida } \\
\text { manual (MM) } \\
\text { Média }\end{array}$} & \multicolumn{2}{|c|}{$\begin{array}{l}\text { Medida compu- } \\
\text { tadorizada (MC) } \\
\text { Média }\end{array}$} & \multicolumn{2}{|c|}{$\begin{array}{c}\text { Comparação } \\
\text { MM e MC } \\
\text { Análise estatística }\end{array}$} \\
\hline & OD & $\mathrm{OE}$ & OD & OE & OD & OE \\
\hline G1 & 9,80 & 9,50 & 9,53 & 9,34 & $\mathrm{p}: 0,12$ & $\mathrm{p}: 0,20$ \\
\hline G2 & 9,28 & 9,10 & 9,38 & 9,27 & $\mathrm{p}: 0,21$ & $\mathrm{p}: 0,19$ \\
\hline G3 & 10,60 & 10,60 & 9,98 & 10,10 & $\mathrm{p}: 0,33$ & $\mathrm{p}: 0,35$ \\
\hline G4 & 8,94 & 8,83 & 8,92 & 8,98 & $\mathrm{p}: 0,33$ & p: 0,19 \\
\hline
\end{tabular}

milimétrica. Em nosso estudo a medida manual foi feita por apenas um médico evitando assim que alguma variação na técnica pudesse interferir no resultado. Outra vantagem da medida digital é ser acessível a qualquer profissional que possua um microcomputador equipado com um programa de captação e edição de imagens, capaz de determinar qualquer medida, podendo ser utilizado como instrumento clínico ou de pesquisa $^{(4)}$. Uma desvantagem desta técnica se comparada com a técnica manual é o custo, relacionado à aquisição dos equipamentos citados anteriormente.

\section{CONCLUSÃO}

Os dados obtidos neste estudo mostram que trabalhos realizados com ambos os métodos podem ser comparados entre si, facilitando a sua aplicação na prática clínica diária.

\section{ABSTRACT}

Purpose: To compare the measurement of the palpebral fissure done either manually and by computer analysis of image of individuals without lid alterations. Methods: One hundred two palpebral fissures from 51 normal subjects have been analyzed. The patients' age ranged from 17 to 84 years, with 25 (49\%) males and $26(51 \%)$ females. Results: There was no statistically significant difference between the measurements done either manually or by computer analysis of images, neither, when we grouped the patients by sex or age. Also we did not observe difference between males and females, and regarding age. Conclusions: The results obtained by both methods are comparable and reliable due to the statistical equivalence of the obtained measurements.

Keywords: Eyelids/anatomy \& histology; Eyelid diseases; Image processing, computer-assisted; Measures; Comparative study

\section{REFERÊNCIAS}

1. Matayoshi S. Anatomia cirúrgica. In: Matayoshi S, Forno E A, Moura E M. Manual de cirurgia plástica ocular. São Paulo: Roca; 2004. p.1-19.

2. Lucci LMD, Portellinha W, Sant'Anna AEBP. Ptose palpebral: estudo de 390 casos. Arq Bras Oftalmol. 1997;60(5):455-7.

3. Pamplona ALF, Osaki MH, Miyazato PY, Sant'Anna AEBP. Média das medidas palpebrais em descendentes de orientais. Arq Bras Oftalmol. 2000; 63(6):469-73.

3. Cruz AA, Coelho RP, Baccega A, Lucchezi MC, Souza AD, Ruiz EE Digital image processing measurement of the upper eyelid contour in Graves disease and congenital blepharoptosis. Ophthalmology. 1998;105(5):913-8.

4. Cruz AAV, Baccega A. Analise Bidimensional computadorizada da fenda palpebral. Arq Bras Oftalmol. 2001;64(1):13-9.

5. Neves LCV, Akaishi PM, Polegato C, Cruz AAV. Efeito da privação de luz no posicionamento da pálpebra superior. Arq Bras Oftalmol. 2004;67(3):455-8.

6. Scion Corporation [home page on the Internet]. Scion Image Beta 4.02. Maryland; [update 2005 Oct 25; cited 2005 Oct 29].: Available from: http: //www.scioncorp.com/frames/fr_scion_products.htm

7. Fox SA. The palpebral fissure. Am J Ophthalmol. 1966;62(1):73-8.

8. Van den Bosch W, Leenders I, Mulder P. Topographic anatomy of the eyelids, and the effects of sex and age. Br J Ophthalmol. 1999;83(3):347-52. 